\title{
Lepton-flavour violating decays in theories with dimension 6 operators
}

\author{
Giovanni Marco Pruna ${ }^{1, a}$ and Adrian Signer ${ }^{1,2, b}$ \\ ${ }^{1}$ Paul Scherrer Institut, $\mathrm{CH}-5232$ Villigen PSI, Switzerland \\ ${ }^{2}$ Physik-Institut, Universität Zürich, $\mathrm{CH}-8057$ Zürich, Switzerland
}

\begin{abstract}
Despite a large experimental effort, so far no evidence for flavour-violating decays of charged leptons such as $l_{i} \rightarrow l_{j} \gamma$ and $l_{i} \rightarrow l_{j} l_{k} l_{k}$ has been found. The absence of a signal puts very severe constraints on many extensions of the Standard Model. Here we apply a model independent approach by studying such decays in the Standard Model effective field theory. Going beyond leading order in the Standard Model couplings and considering all dimension 6 operators that might lead to lepton-flavour violation, we are able to extract limits on a large number of Wilson coefficients of such operators. We are also able to compare the impact of particular searches and find, for example, that flavour-violating decays of the $Z$-boson $Z \rightarrow \mu e$ are much more constrained from low-energy experiments $\mu \rightarrow e \gamma$ than from the limits of current and future direct searches at high energy.
\end{abstract}

\section{Introduction}

It is well established that the Standard Model (SM) of particle physics provides a structure of gauge symmetries that accidentally conserves the leptonic flavour. This opens up a possibility for very stringent tests of the SM and powerful searches for physics beyond the SM. For this reason, a longstanding experimental effort has been devoted to the search of lepton-flavour violating (LFV) signals both in the neutral and charged sectors.

In the neutrino sector, the evidence for flavour violation is by now established beyond any doubt [1-3]. Thus, the SM with only left-handed neutrinos needs to be extended. Concerning the charged lepton sector, searches for LFV in $\mu$ and $\tau$ decays at low energy experiments as well as in $Z$ boson decays at high energy experiments have been analysed for decades, resulting in ever more stringent limits on various LFV branching ratios (BRs) of the aforementioned particles. One possibility to study and interpret the absence (so far) of any charged LFV signals is to consider specific models beyond the SM and a large effort has been made in this direction (see e.g., [4-10]).

In this proceeding, the adopted strategy follows from a bottom-up approach established in [11] and refined in the past thirty years $[12,13]$. The SM is considered to be an effective theory valid up to an (unknown) large energy scale $\Lambda$ and the Lagrangian consists of a systematic dimensional expansion in $1 / \Lambda$ that includes all operators constructed from SM fields (without right-handed neutri-

\footnotetext{
a e-mail: Giovanni-Marco.Pruna@psi.ch

be-mail: Adrian.Signer@psi.ch
}

nos) that respect the SM gauge symmetries, i.e.

$$
\mathcal{L}=\mathcal{L}_{\mathrm{SM}}+\frac{1}{\Lambda} \sum_{k} C_{k}^{(5)} Q_{k}^{(5)}+\frac{1}{\Lambda^{2}} \sum_{k} C_{k}^{(6)} Q_{k}^{(6)}+O\left(\frac{1}{\Lambda^{3}}\right)
$$

The coefficients $C_{k}^{(d)}$ are couplings and, very much like the usual couplings of the SM, they have to be determined by experiment. Beyond tree level, a renormalisation scheme has to be chosen to give the couplings a welldefined meaning.

Once an ultraviolet (UV) complete theory is known that describes the effects of physics beyond the scale $\Lambda$, the coefficients $C_{k}^{(d)}$ of the Lagrangian can be expressed in terms of the parameters of the UV complete theory. Such a matching has to be done at the scale $\Lambda$. To obtain the coefficients at the relevant low energy scale, renormalisationgroup (RG) techniques have to be used to determine the running. We want to stress that due to operator mixing the $\mathrm{RG}$ evolution leads to qualitatively new effects and, at least for the time being, is not primarily a question of precision.

Performing an expansion in $1 / \Lambda$ of the most general gauge-invariant Lagrangian one is allowed to write only one Dim-5 operator [14-16]. This operator provides both neutrino mass terms and LFV in the neutrino sector. Going beyond tree level, it also causes LFV in the charged sector. However, the smallness of the neutrino masses results in very severe constraints on its coefficients. These constraints in turn imply that the amount of charged LFV provided by the Dim-5 operator is far below any foreseeable experimental capability. Hence, the next step consists both in the scrutiny of the 19 Dim- 6 operators that yield LFV and in the interpretation of the current BR limits with respect to such extended parameter space. Given the complete lack of information on the structure of the 
UV complete theory, we make no assumption whatsoever on the relative importance of the various Dim- 6 operators.

In the literature, muon decays are often studied using an effective theory that stops one step before, by integrating out any dynamics at the electroweak scale. The resulting effective theory is simpler and contains fewer parameters. However, we refrain from doing this and work directly with the Lagrangian of Eq. (1). This provides us with a framework to combine constraints from low-energy experiments such as $\mu^{+} \rightarrow e^{+} \gamma$ and $\mu^{+} \rightarrow e^{+} e^{-} e^{+}$with LFV searches at higher energies, such as LFV decays of the $Z$ boson or $H \rightarrow \tau \mu$.

On the low energy side, very accurate limits on the LFV muon decays $\mu^{+} \rightarrow e^{+} \gamma$ and $\mu^{+} \rightarrow e^{+} e^{-} e^{+}$have been made available by the MEG [17] and SINDRUM [18] collaborations, respectively; in the near future, an upgrade of the MEG experiment [19] will take place almost in parallel with Mu3e, a new experiment devoted to the $\mu \rightarrow 3 e$ searches [20]. In the tauonic sector, a big effort is ongoing to continuously improve the bounds on several decay channels in various experiments [21, 22].

On the high energy side, interesting searches have been performed at LEP in the context of LFV decays of the $Z$ boson [23-26], and further improvements have either been made or are expected from the ATLAS [27] and CMS [28] experiments at the LHC collider. Moreover, after the discovery of the Higgs boson [29, 30], new limits on the $H \rightarrow \tau \mu \mathrm{BR}$ are currently under investigation [31, 32].

In this proceeding, the constraints on various Dim-6 coefficients are reviewed in light of the latest bounds on LFV $\mu$ and $\tau$ decays and in the assumption that the relevant operators are not correlated. Due to the very good accuracy of the bounds on $l_{i} \rightarrow l_{j} \gamma$ transitions, these processes will be considered at the one-loop level, while the rest of our study will be performed at the tree level. The tables supplied in this note are neither meant to be complete nor to exhaust the general discussion on LFV decays in theories with Dim-6 operators. For example LFV transitions in nuclei, where a substantial improvement [33, 34] in the experimental sensitivity with respect to the current best limit [35] is expected, is not considered. At this stage, we also do not include decays $l_{i} \rightarrow l_{j} 2 l_{k}$ with $k \neq j$. Furthermore, we also neglect correlations among operators and potentially important two loop Barr-Zee effects. The main two messages that will be delivered in the following sections are: (i) the richness of the information on the coefficients $C_{k}^{(d)}$ that can be gained from low-energy experiments if Eq. (1) is taken seriously as a quantum field theory and (ii) the fact that the limits on LFV decays of the $Z$ boson from low-energy experiments are far more stringent than those from the high-energy experiments.

Lastly, a remark is required: these proceedings are largely based on the elements collected in Ref. [36], to which the reader is redirected for further details.

\section{LFV dimension 6 operators}

Among the 19 Dim-6 operators that trigger LFV transitions, the relevant ones for the study of $l_{i} \rightarrow l_{j} \gamma$ (at the one loop level), $l_{i} \rightarrow 3 l_{j}, Z \rightarrow l_{i} l_{j}$ and $H \rightarrow l_{i} l_{j}$ are collected in Table 1.

\begin{tabular}{|c|c|c|c|}
\hline \multicolumn{2}{|r|}{$\psi^{2} X \varphi$} & \multicolumn{2}{|r|}{$\psi^{2} \varphi^{2} D$} \\
\hline$Q_{e W}$ & $\left(\bar{l}_{p} \sigma^{\mu v} e_{r}\right) \tau^{I} \varphi W_{\mu v}^{I}$ & $Q_{\varphi l}^{(1)}$ & $\left(\varphi^{\dagger} i \stackrel{\leftrightarrow}{D_{\mu}} \varphi\right)\left(\bar{l}_{p} \gamma^{\mu} l_{r}\right)$ \\
\hline \multirow[t]{2}{*}{$Q_{e B}$} & $\left(\bar{l}_{p} \sigma^{\mu v} e_{r}\right) \varphi B_{\mu v}$ & $Q_{\varphi l}^{(3)}$ & $\left(\varphi^{\dagger} i \stackrel{\leftrightarrow}{D_{\mu}^{I}} \varphi\right)\left(\bar{l}_{p} \tau^{I} \gamma^{\mu} l_{r}\right)$ \\
\hline & & $Q_{\varphi e}$ & $\left(\varphi^{\dagger} i \stackrel{\leftrightarrow}{D}_{\mu} \varphi\right)\left(\bar{e}_{p} \gamma^{\mu} e_{r}\right)$ \\
\hline \multicolumn{4}{|c|}{$\psi^{2} \varphi^{3}$} \\
\hline \multicolumn{2}{|r|}{$Q_{e \varphi}$} & \multicolumn{2}{|r|}{$\left(\varphi^{\dagger} \varphi\right)\left(\bar{l}_{p} e_{r} \varphi\right)$} \\
\hline \multicolumn{2}{|r|}{$(\overline{l l})(\overline{l l})$} & \multicolumn{2}{|r|}{$(\overline{l l})(\bar{q} q)$} \\
\hline$Q_{l l}$ & $\left(\bar{l}_{p} \gamma_{\mu} l_{r}\right)\left(\overline{l_{s}} \gamma^{\mu} l_{t}\right)$ & $Q_{\text {lequ }}^{(1)}$ & $\left(\overline{l_{p}^{j}} e_{r}\right) \varepsilon_{j k}\left(\bar{q}_{s}^{k} u_{t}\right)$ \\
\hline$Q_{e e}$ & $\left(\bar{e}_{p} \gamma_{\mu} e_{r}\right)\left(\overline{e_{s}} \gamma^{\mu} e_{t}\right)$ & $Q_{\text {lequ }}^{(3)}$ & $\left(\overrightarrow{l_{p}^{j}} \sigma_{\mu \nu} e_{r}\right) \varepsilon_{j k}\left(\bar{q}_{s}^{k} \sigma^{\mu \nu} u_{t}\right)$ \\
\hline$Q_{l e}$ & $\left(\bar{l}_{p} \gamma_{\mu} l_{r}\right)\left(\bar{e}_{s} \gamma^{\mu} e_{t}\right)$ & & \\
\hline
\end{tabular}

Table 1. LFV Dim-6 operators.

Working in the physical basis rather than in the gauge basis, the dipole operators (set $\psi^{2} X \varphi$ ) are rewritten using

$$
\begin{aligned}
Q_{e B} & \rightarrow Q_{e \gamma} c_{W}-Q_{e Z} s_{W}, \\
Q_{e W} & \rightarrow-Q_{e \gamma} s_{W}-Q_{e Z} c_{W},
\end{aligned}
$$

where $s_{W}=\sin \left(\theta_{W}\right)$ and $c_{W}=\cos \left(\theta_{W}\right)$ are the sine and cosine of the weak mixing angle. The term

$$
\mathcal{L}_{e \gamma} \equiv \frac{C_{e \gamma}}{\Lambda^{2}} Q_{e \gamma}+\text { h.c. }=\frac{C_{e \gamma}^{p r}}{\Lambda^{2}}\left(\bar{l}_{p} \sigma^{\mu v} e_{r}\right) \varphi F_{\mu v}+\text { h.c. }
$$

where $F_{\mu \nu}$ is the electromagnetic field-strength tensor, is then the only term in the Dim-6 Lagrangian that induces a $l_{i} \rightarrow l_{j} \gamma$ transition at tree level.

In the Feynman gauge, the combination of $Q_{e \varphi}$ with the Dim-4 SM Yukawa terms gives

$$
\begin{aligned}
& \mathcal{L}_{\text {Yukawa }}+\mathcal{L}_{e \varphi}= \\
= & \frac{v}{\sqrt{2}}\left(-y_{p r}+\frac{v^{2}}{2 \Lambda^{2}} C_{e \varphi}^{p r}\right) \bar{e}_{p} e_{r} \\
+ & \frac{1}{\sqrt{2}}\left(-y_{p r}+\frac{v^{2}}{2 \Lambda^{2}} C_{e \varphi}^{p r}\right) \bar{e}_{p} e_{r} h+\frac{v^{2}}{\sqrt{2} \Lambda^{2}} C_{e \varphi}^{p r} \bar{e}_{p} e_{r} h \\
+ & \frac{i}{\sqrt{2}}\left(-y_{p r}+\frac{v^{2}}{2 \Lambda^{2}} C_{e \varphi}^{p r}\right) \bar{e}_{p} e_{r} \widehat{Z} \\
+ & i\left(-y_{p r}+\frac{v^{2}}{2 \Lambda^{2}} C_{e \varphi}^{p r}\right) \bar{e}_{p} v_{r} \widehat{W}^{+}+[\ldots] .
\end{aligned}
$$

From Eq. (5), it is understood that any 3-point off-diagonal interaction involving Goldstone bosons is not physical, i.e. it can be removed by an orthogonal transformation ${ }^{1}$. However, this procedure leaves a residual term with a physical Higgs supporting LFV currents, which is the only treelevel contribution to the $H \rightarrow l_{i} l_{j}$ transition.

\footnotetext{
${ }^{1}$ In this proceeding, any impact of such diagonalisation on the charged lepton eigenstates and their masses will be neglected.
} 
Concerning the decay $Z \rightarrow l_{i} l_{j}$, such transition is triggered at the tree level by the following operators: $Q_{e Z}$ (the dipole contribution), $Q_{\varphi l}^{(1)}, Q_{\varphi l}^{(3)}$ and $Q_{\varphi e}$. In addition, $\mu$ and $\tau$ three body decays are also produced by the point-like four-fermion operators $Q_{l l}, Q_{l e}$ and $Q_{e e}$.

The impact of $Q_{\text {lequ }}^{(1)}$ and $Q_{\text {lequ }}^{(3)}$ in the $l_{i} \rightarrow l_{j} \gamma$ transition is of a different kind: they contribute in the running of the dipole operators, hence they must be carefully included when different energy scales are considered, as was shown in [36-39].

\section{Branching Ratios}

In the limit where $m_{1} \gg m_{2}$ and no correlation is considered among different operators, the partial widths of the aforementioned processes are the following:

- for the two-body decay $l_{1}^{ \pm} \rightarrow l_{2}^{ \pm} \gamma$, one has

$$
\Gamma\left(l_{1}^{ \pm} \rightarrow l_{2}^{ \pm} \gamma\right)=\frac{m_{1}^{3}}{4 \pi \Lambda^{4}}\left(\left|C_{T L}\right|^{2}+\left|C_{T R}\right|^{2}\right),
$$

where the contributions to $C_{T L}$ and $C_{T R}$ (at the energy scale $\lambda=m_{Z}$, in the assumption that the coefficients are real) are given in Table 2, according to [36] (see also [40]). The coefficients $C_{k}^{(d)}$ in Table 2 are to be interpreted as renormalised in the $\overline{\mathrm{MS}}$-scheme;

- for the three-body decay $l_{1}^{ \pm} \rightarrow l_{2}^{ \pm} l_{2}^{\mp} l_{2}^{ \pm}$, one has

$$
\begin{aligned}
& \Gamma\left(l_{1}^{+} \rightarrow l_{2}^{+} l_{2}^{-} l_{2}^{+}\right)=\left(40 e^{2} v^{2}\left(\left|C_{e \gamma}^{12}\right|^{2}+\left|C_{e \gamma}^{21}\right|^{2}\right)\left(8 \ln \left[\frac{m_{1}}{m_{2}}\right]-11\right)\right. \\
& +\frac{2 m_{1}^{4}}{m_{Z}^{2}}\left(\left(5-20 s_{W}^{2}+36 s_{W}^{4}\right)\left|C_{e Z}^{12}\right|^{2}\right. \\
& \left.\quad+4\left(1-4 s_{W}^{2}+9 s_{W}^{4}\right)\left|C_{e Z}^{21}\right|^{2}\right) \\
& +\frac{15 m_{2}^{2} m_{1}^{2} v^{2}\left(\left|C_{e \varphi}^{12}\right|^{2}+\left|C_{e \varphi}^{21}\right|^{2}\right)}{8 m_{H}^{4}} \\
& +10 m_{1}^{2}\left(1-4 s_{W}^{2}+12 s_{W}^{4}\right)\left|C_{\varphi e}^{12}\right|^{2} \\
& +20 m_{1}^{2}\left(1-4 s_{W}^{2}+6 s_{W}^{4}\right)\left(\left|C_{\varphi l(1)}^{12}\right|^{2}+\left|C_{\varphi l(3)}^{12}\right|^{2}\right)+ \\
& +10 m_{1}^{2}\left(\left|C_{l e}^{1112}\right|^{2}+\left|C_{l e}^{1211}\right|^{2}\right) \\
& \left.+80 m_{1}^{2}\left(\left|C_{e e}^{1112}\right|^{2}+\left|C_{l l}^{1112}\right|^{2}\right)\right) \frac{m_{1}^{3}}{30(8 \pi)^{3} \Lambda^{4}},
\end{aligned}
$$

where the integration over the phase space of the photonic dipole contribution gives rise to a logarithmic term, in agreement with [41]. The result in Eq. (7) is also valid for $\Gamma\left(l_{1}^{-} \rightarrow l_{2}^{-} l_{2}^{+} l_{2}^{-}\right)$;

- flavour-violating $Z$ decays can be parametrised at the tree level by means of the following four operators:

$$
\begin{aligned}
& \Gamma\left(Z \rightarrow l_{1}^{ \pm} l_{2}^{\mp}\right)=\frac{m_{Z}^{3} v^{2}}{12 \pi \Lambda^{4}}\left(\left|C_{e Z}^{12}\right|^{2}+\left|C_{e Z}^{21}\right|^{2}\right. \\
& \left.+\left|C_{\varphi e}^{12}\right|^{2}+\left|C_{\varphi l(1)}^{12}\right|^{2}+\left|C_{\varphi l(3)}^{12}\right|^{2}\right),
\end{aligned}
$$

and as one can see from the last equation, all of their contributions occur at the same order. In Eq. (8) we have summed over the two possible final states, $l_{1}^{+} l_{2}^{-}$and $l_{1}^{-} l_{2}^{+}$;
- for the Higgs boson decay $H \rightarrow l_{1}^{ \pm} l_{2}^{\mp}$, one has

$$
\Gamma\left(H \rightarrow l_{1}^{ \pm} l_{2}^{\mp}\right)=\frac{m_{H} v^{4}}{16 \pi \Lambda^{4}}\left(\left|C_{e \varphi}^{12}\right|^{2}+\left|C_{e \varphi}^{21}\right|^{2}\right),
$$

where only one operator contributes at tree level. As for the $Z$ decays, in Eq. (9) we have summed over the two possible decays $l_{1}^{+} l_{2}^{-}$and $l_{1}^{-} l_{2}^{+}$.

\begin{tabular}{||c|c||}
\hline \hline Op. & $C_{T L}$ or $C_{T R}(1 \longleftrightarrow 2)$ \\
\hline$Q_{e \gamma}$ & $-C_{e \gamma}^{12} \frac{v}{\sqrt{2}}$ \\
$Q_{e Z}$ & $-C_{e Z}^{12} \frac{e m_{Z}}{16 \sqrt{2} \pi^{2}}\left(3-6 c_{W}^{2}+4 c_{W}^{2} \log \left[c_{W}^{2}\right]\right)$ \\
$Q_{\varphi l}^{(1)}$ & $-C_{\varphi l(1)}^{12} \frac{e m_{1}\left(1+s_{W}^{2}\right)}{24 \pi^{2}}$ \\
$Q_{\varphi l}^{(3)}$ & $C_{\varphi l(3)}^{12} \frac{e m_{1}\left(3-2 s_{W}^{2}\right)}{48 \pi^{2}}$ \\
$Q_{\varphi e}$ & $C_{\varphi e}^{12} \frac{e m_{2}\left(3-2 s_{W}^{2}\right)}{48 \pi^{2}}$ \\
$Q_{e \varphi}$ & $C_{e \varphi}^{12} \frac{e v \operatorname{Max}\left(m_{1}^{2}, m_{2}^{2}\right)}{96 \sqrt{2} m_{H}^{2} \pi^{2}}\left(4+3 \log \left[\frac{\operatorname{Max}\left(m_{1}^{2}, m_{2}^{2}\right)}{m_{H}^{2}}\right]\right)$ \\
$Q_{l e}$ & $\frac{e}{16 \pi^{2}} \sum_{i=e, \mu, \tau} m_{i} C_{l e}^{1 i i 2}$ \\
\hline \hline
\end{tabular}

Table 2. Leading order results (up to one loop) for the contributions of the various (real) Dim-6 operators to the $l_{1} \rightarrow l_{2} \gamma$ decay at the energy scale $\lambda=m_{Z}$.

In order to numerically evaluate the BRs, the Particle Data Group (PDG) values [42] for the physical constants and for the $\mu$ and $\tau$ total decay width were used.

\section{Results}

Here, the elements collected in Section 3 are combined with the experimental limits on LFV transitions at high and low energies. Since the category of transitions $l_{i} \rightarrow l_{j} \gamma$ was studied at the one-loop level, the limits on $\mu \rightarrow e \gamma$ and $\tau \rightarrow \mu \gamma$ from MEG and BaBar were interpreted at the energy scale $\lambda=m_{Z}$ by means of the QED running of the Wilson coefficients in Eq. (6). Being absolutely rigorous, also the limits on three-body decays should be studied at the one-loop level and reinterpreted at the $Z$-boson mass scale. However, it was proved by direct computation that such running, in absence of correlations among operators, only affects the limits at the $10 \%$ level. Hence it is not relevant in the following discussion where differences of orders of magnitude are involved.

\subsection{Limits at a fixed energy $\lambda=m_{Z}$}

Under the assumption that only one operator at a time is non-vanishing and the corresponding coefficients are real, the numerical limits of Tables 3-4 are obtained. Note 
that $C_{\varphi e}^{21}=\left(C_{\varphi e}^{12}\right)^{*} \doteq C_{\varphi e}^{12}$ (and similar for $C_{\varphi l(1)}$ and $\left.C_{\varphi l(3)}\right)$ since we treat the coefficients as real. We also have $C_{e e}^{p r s t}=C_{e e}^{s t p r}=\left(C_{e e}^{t s r p}\right)^{*} \doteq C_{e e}^{t s r p}$ (and similar for $C_{l l}$ ), where again the last step assumes the coefficients to be real.

\begin{tabular}{||c||c|c|c||}
\hline \hline $\begin{array}{c}\text { Coeff. } \\
\lambda=m_{Z}\end{array}$ & $\begin{array}{c}\mu^{+} \rightarrow e^{+} \gamma \\
\mathrm{BR} \leq 5.7 \cdot 10^{-13}\end{array}$ & $\begin{array}{c}Z \rightarrow e^{ \pm} \mu^{\mp} \\
\mathrm{BR} \leq 7.5 \cdot 10^{-7}\end{array}$ & $\begin{array}{c}\mu^{+} \rightarrow e^{+} e^{-} e^{+} \\
\mathrm{BR} \leq 1.0 \cdot 10^{-12}\end{array}$ \\
\hline$C_{e \gamma}^{21 / 12}$ & $2.5 \cdot 10^{-16}$ & & $3.8 \cdot 10^{-15}$ \\
$C_{e Z}^{21 / 12}$ & $1.4 \cdot 10^{-13}$ & $3.9 \cdot 10^{-8}$ & $4.0 \cdot 10^{-8}$ \\
$C_{\varphi l(1)}^{12}$ & $2.6 \cdot 10^{-10}$ & $3.9 \cdot 10^{-8}$ & $3.5 \cdot 10^{-11}$ \\
$C_{\varphi l(3)}^{12}$ & $2.5 \cdot 10^{-10}$ & $3.9 \cdot 10^{-8}$ & $3.5 \cdot 10^{-11}$ \\
$C_{\varphi e}^{12}$ & $2.5 \cdot 10^{-10}$ & $3.9 \cdot 10^{-8}$ & $3.7 \cdot 10^{-11}$ \\
$C_{e \varphi}^{21 / 12}$ & $2.8 \cdot 10^{-8}$ & & $8.7 \cdot 10^{-6}$ \\
$C_{l e}^{2111 / 1112}$ & $4.4 \cdot 10^{-8}$ & & $3.1 \cdot 10^{-11}$ \\
$C_{l e}^{2221 / 1222}$ & $2.1 \cdot 10^{-10}$ & & \\
$C_{l e}^{2331 / 1332}$ & $1.2 \cdot 10^{-11}$ & & $1.1 \cdot 10^{-11}$ \\
$C_{e e}^{2111}$ & \multicolumn{3}{|l}{} \\
$C_{l l}^{2111}$ & & & $1.1 \cdot 10^{-11}$ \\
\hline \hline
\end{tabular}

Table 3. Limits on the Wilson coefficients in $[\mathrm{GeV}]^{-2}$ contributing to the LFV muonic transitions. $\mu \rightarrow 3 e$ will lead to better limits on $C_{e Z}$ once NLO corrections are taken into account.

Focusing on LFV muonic transitions, the first thing to notice in Table 3 is that the best limits are currently always obtained from the constraints established by low energy experiments. The constraints from LFV decays $Z \rightarrow e^{ \pm} \mu^{\mp}$ on the coefficients of the operators $Q_{e Z}, Q_{\varphi l(1,3)}$ and $Q_{\varphi e}$ are considerably less stringent than from $\mu^{+} \rightarrow e^{+} \gamma$ and $\mu^{+} \rightarrow e^{+} e^{-} e^{+}$.

Even if at a future high energy lepton colliders (e.g. FCC-ee [43]) the LEP limits for $\operatorname{BR}\left(Z \rightarrow e^{ \pm} \mu^{\mp}\right)$ could be improved by several orders of magnitude it is still the case that the information one can get from such future machine on $Z \rightarrow e^{ \pm} \mu^{\mp}$ is only complementary, and surely never competitive with the planned future low energy experiments. Indeed, if no correlation is assumed, from Eq. (8) one deduces that in order to reach a probing power equivalent to the current low energy experiments, the required branching ratios for $Z \rightarrow e^{ \pm} \mu^{\mp}$ are $\mathrm{BR} \leq 9.5 \cdot 10^{-18}$ for $C_{e Z}^{21}$, $\mathrm{BR} \leq 5.9 \cdot 10^{-13}$ for $C_{\varphi l(1,3)}^{21}$ and $\mathrm{BR} \leq 6.6 \cdot 10^{-13}$ for $C_{\varphi e}^{21}$, respectively. Hence, the operator $Q_{e Z}^{21}$ will be out of the reach of FCC-ee, while the coefficients of $Q_{\varphi l(1)}^{12}, Q_{\varphi l(3)}^{12}$ and $Q_{\varphi e}^{12}$ could be constrained with (more or less) the same precision of the dated SINDRUM experiment. The interplay between a high-luminosity $Z$-factory and low-energy experiments has also been studied with concrete BSM models (see e.g. [44]).

Comparing the limits obtained from $\mu^{+} \rightarrow e^{+} \gamma$ with $\mu^{+} \rightarrow e^{+} e^{-} e^{+}$it is clear that both experiments provide very valuable information. The standard statement is that $\mu \rightarrow 3 e$ is more powerful in constraining four-fermion operators (contact interactions) whereas $\mu \rightarrow e \gamma$ is more sensitive to the dipole operators. However, a closer look reveals that this is only partially true. Some four-fermion operators have stringent limits due to $\mu \rightarrow e \gamma$ (at one loop) but are not constrained by $\mu \rightarrow 3 e$ (at tree level). Furthermore, in the long term $\mu \rightarrow 3 e$ might well be competitive in putting limits on the photon dipole operator. The BR for $\mu \rightarrow 3 e$ is expected to be improved by several orders of magnitude. A limit $\operatorname{BR}(\mu \rightarrow 3 e) \leq 4 \cdot 10^{-15}$ is as constraining to $C_{e \gamma}^{21}$ as the current $\operatorname{BR}(\mu \rightarrow e \gamma) \leq 5.7 \cdot 10^{-13}$. Of course, the BR for $\mu \rightarrow e \gamma$ is also expected to improve and in the longer term, there will also be very strong constraints from future muon conversion experiments. Regarding the operators with a Higgs, $\mu \rightarrow 3 e$ provides the stronger limits except for $C_{e \varphi}$. However, even stronger limits on this coefficient can be obtained by looking at muon conversion $[45,46]$. Let us mention again that our limit on $C_{e \varphi}$ is indicative at best, since we have not taken into account numerically important two-loop contributions from Barr-Zee diagrams [47, 48]. We will come back to this when discussing tau decays.

Note that the limits for $C_{l l}^{2111}$ and $C_{e e}^{2111}$ differ by a factor 2 from the corresponding limits given in [49], since we write the terms containing $Q_{l l}$ (and $Q_{e e}$ ) in the Lagrangian as

$$
\begin{aligned}
\mathcal{L}_{l l}= & \sum_{p r s t} \frac{C_{l l}^{p r s t}}{\Lambda^{2}}\left(\bar{l}_{p} \gamma_{v} l_{r}\right)\left(\bar{l}_{s} \gamma^{v} l_{t}\right) \\
& =2 \frac{C_{l l}^{211}}{\Lambda^{2}}\left(\bar{l}_{2} \gamma_{v} l_{1}\right)\left(\bar{l}_{1} \gamma^{v} l_{1}\right)+[\ldots],
\end{aligned}
$$

where we exploited the symmetry of the operator.

\begin{tabular}{||c||c|c|c||}
\hline \hline $\begin{array}{c}\text { Coeff. } \\
\lambda=m_{Z}\end{array}$ & $\begin{array}{c}\tau^{+} \rightarrow \mu^{+} \gamma \\
\mathrm{BR} \leq 4.4 \cdot 10^{-8}\end{array}$ & $\begin{array}{c}Z \rightarrow \mu^{ \pm} \tau^{\mp} \\
\mathrm{BR} \leq 1.2 \cdot 10^{-5}\end{array}$ & $\begin{array}{c}\tau^{+} \rightarrow \mu^{+} \mu^{-} \mu^{+} \\
\mathrm{BR} \leq 2.1 \cdot 10^{-8}\end{array}$ \\
\hline$C_{e \gamma}^{32 / 23}$ & $2.7 \cdot 10^{-12}$ & & $3.8 \cdot 10^{-11}$ \\
$C_{e Z}^{32 / 23}$ & $1.5 \cdot 10^{-9}$ & $1.5 \cdot 10^{-7}$ & $8.7 \cdot 10^{-7}$ \\
$C_{\varphi l(1)}^{23}$ & $1.7 \cdot 10^{-7}$ & $1.5 \cdot 10^{-7}$ & $1.3 \cdot 10^{-8}$ \\
$C_{\varphi l(3)}^{23}$ & $1.6 \cdot 10^{-7}$ & $1.5 \cdot 10^{-7}$ & $1.3 \cdot 10^{-8}$ \\
$C_{\varphi e}^{23}$ & $1.6 \cdot 10^{-7}$ & $1.5 \cdot 10^{-7}$ & $1.3 \cdot 10^{-8}$ \\
\cline { 1 - 1 } $\begin{array}{c}\text { Coeff. } \\
\lambda=m_{Z}\end{array}$ & & $H \rightarrow \mu^{ \pm} \tau^{\mp}$ & \\
\cline { 1 - 1 }$C_{e \varphi}^{32 / 23}$ & $1.9 \cdot 10^{-6}$ & $9.0 \cdot 10^{-8}$ & $1.6 \cdot 10^{-5}$ \\
$C_{l e}^{3112 / 2113}$ & $4.8 \cdot 10^{-4}$ & & \\
$C_{l e}^{3222 / 2223}$ & $2.3 \cdot 10^{-6}$ & & $1.1 \cdot 10^{-8}$ \\
$C_{l e}^{3222 / 2223}$ & $1.4 \cdot 10^{-7}$ & & \\
$C_{e e}^{3222}$ & & & $4.0 \cdot 10^{-9}$ \\
$C_{l l}^{3222}$ & & & $4.0 \cdot 10^{-9}$ \\
\hline \hline
\end{tabular}

Table 4. Limits on the Wilson coefficients in $[\mathrm{GeV}]^{-2}$ contributing to the LFV tauonic transitions.

The analysis can easily be adapted to the tau sector and the corresponding limits on the coefficients are given in Table 4. We should note that similar results can be found 
in the literature $[40,50-52]$ and a review of studies of the tauonic limits can be found e.g. in [49]. The pattern is very similar to muonic decays. However, the limits from LFV $Z$ decays play a more important role and if they were to be improved by several orders of magnitude at a future high energy lepton colliders, they could provide the most stringent limits on $C_{\varphi l(1)}^{23}, C_{\varphi l(3)}^{23}$ and $C_{\varphi e}^{23}$.

A new feature of the tau sector is the appearance of the $H \rightarrow \tau \mu$ decay, which has caused a huge theoretical activity [53-59]. At tree level, the only operator that induces such a LFV decay in the Lagrangian Eq. (1) is $Q_{e \varphi}^{32 / 23}$. The non-observation of this decay at the LHC is placing a limit on the $C_{e \varphi}^{32 / 23}$ coefficient that is more stringent then any limit extracted from the low energy experiment. This decay has been considered extensively in the literature, often with phenomenologically motivated Lagrangians [53], where the LFV interactions are given in terms of generalised Yukawa matrices. In terms of Dim-6 operators, the deviations from the SM Yukawa interactions are induced by the operator $Q_{e \varphi}$, as can be seen in Eq. (5). Our limits on $C_{e \varphi}^{32 / 23}$ in Table 4 indicate that $H \rightarrow \tau \mu$ provides the strongest constraint, followed by $\tau \rightarrow \mu \gamma$ and $\tau \rightarrow 3 \mu$. This is in qualitative agreement with [54]. However, we should mention once more that the limits extracted here from low-energy observables do not consider the impact of Barr-Zee type two-loop contributions, which is particularly important in this case [54].

In Table 4, the ATLAS \& CMS data on $H \rightarrow \tau \mu$ are considered only in their function of placing new limits on such a transition. However, if one wants to consider the possibility that the picture corresponds to a signal compatible with $\mathrm{BR}(\mathrm{H} \rightarrow \tau \mu)=0.84 \%$ (with a significance of $2.4 \sigma$ ), then a trivial computation leads to $C_{e \varphi}^{32 / 23} / \Lambda^{2} \sim 8.7 \cdot 10^{-8} \mathrm{GeV}^{-2}$ or, in the same way, to $\Lambda \sim 3.4 \sqrt{C_{e \varphi}^{32 / 23}}$ TeV. From this, it follows that an effective coefficient that preserves the perturbative behaviour $C_{e \varphi}^{32 / 23} \leq 1$ would imply a limit on the UV completion scale $\Lambda \geq 3.4 \mathrm{TeV}$, that in turn preserves the perturbative behaviour of the expansion in Eq. (1), being $C_{e \varphi}(v / \Lambda)^{2} \leq$ $O\left(10^{-2}\right)$. Here, no further speculation will be carried out about the possible nature of the UV completion and we refer to e.g. $[60,61]$ for a deeper discussion on the topic.

\subsection{Limits from RGE}

In the previous subsection we have given limits that were obtained by comparing NLO (or LO) calculations using Eq. (1) to experimental limits on branching ratios. However, the most direct link of the Wilson coefficients $C_{k}^{(d)}$ of Eq. (1) to the (unknown) UV complete theory is at the large scale $\Lambda$. In order to obtain the Wilson coefficients at the low scale $\left(m_{Z}\right.$ or $\left.m_{l}\right)$ that are relevant for the branching ratios, $\mathrm{RG}$ evolution has to be applied. This has two effects. First, the limits on $C_{k}^{(d)}\left(m_{Z}\right)$ will be translated into limits $C_{k}^{(d)}(\Lambda)$. Second, and more important, qualitatively new effects happen. It might well be possible, that a certain UV complete theory does result in a vanishing coefficient $C_{e \gamma}(\Lambda)=0$ at the large scale. Nevertheless, the corresponding theory can lead to a LFV decay $l^{i} \rightarrow l^{j} \gamma$. In fact, other operators that are generated by the UV-complete theory at $\Lambda$ can mix under RG evolution with $Q_{e \gamma}$ and result in a non-vanishing Wilson coefficient $C_{e \gamma}\left(m_{z}\right) \neq 0$.

In principle, the complete set of Dim-6 operators has to be taken into account to study $C_{e \gamma}\left(m_{z}\right)$. Restricting ourselves to operators that either directly or indirectly mix into $Q_{e \gamma}$, the RG evolution of the corresponding coefficients is given in [36]:

$$
\begin{aligned}
16 \pi^{2} \frac{\partial C_{e \gamma}^{i j}}{\partial \log \lambda} & \simeq\left(\frac{47 e^{2}}{3}+\frac{e^{2}}{4 c_{W}^{2}}-\frac{9 e^{2}}{4 s_{W}^{2}}+3 Y_{t}^{2}\right) C_{e \gamma}^{i j} \\
& +6 e^{2}\left(\frac{c_{W}}{s_{W}}-\frac{s_{W}}{c_{W}}\right) C_{e Z}^{i j}+16 e Y_{t} C_{i j 33}^{(3)}, \\
16 \pi^{2} \frac{\partial C_{e Z}^{i j}}{\partial \log \lambda} & \simeq-\frac{2 e^{2}}{3}\left(\frac{2 c_{W}}{s_{W}}+\frac{31 s_{W}}{c_{W}}\right) C_{e \gamma}^{i j} \\
& +2 e\left(\frac{3 c_{W}}{s_{W}}-\frac{5 s_{W}}{c_{W}}\right) Y_{t} C_{i j 33}^{(3)} \\
& +\left(-\frac{47 e^{2}}{3}+\frac{151 e^{2}}{12 c_{W}^{2}}-\frac{11 e^{2}}{12 s_{W}^{2}}+3 Y_{t}^{2}\right) C_{e Z}^{i j}, \\
16 \pi^{2} \frac{\partial C_{i j 33}^{(3)}}{\partial \log \lambda} & \simeq \frac{7 e Y_{t}}{3} C_{e \gamma}^{i j}+\frac{e Y_{t}}{2}\left(\frac{3 c_{W}}{s_{W}}-\frac{5 s_{W}}{3 c_{W}}\right) C_{e Z}^{i j}+ \\
& +\left(\frac{2 e^{2}}{9 c_{W}^{2}}-\frac{3 e^{2}}{s_{W}^{2}}+\frac{3 Y_{t}^{2}}{2}+\frac{8 g_{S}^{2}}{3}\right) C_{i j 33}^{(3)} \\
& +\frac{e^{2}}{8}\left(\frac{5}{c_{W}^{2}}+\frac{3}{s_{W}^{2}}\right) C_{i j 33}^{(1)}, \\
& +\left(-\frac{11 e^{2}}{3 c_{W}^{2}}+\frac{15 Y_{t}^{2}}{2}-8 g_{S}^{2}\right) C_{i j 33}^{(1)} . \\
16 \pi^{2} \frac{\partial C_{i j 33}^{(1)}}{\partial \log \lambda} & \simeq\left(\frac{30 e^{2}}{c_{W}^{2}}+\frac{18 e^{2}}{s_{W}^{2}}\right) C_{i j 33}^{(3)} \\
& (11) \\
&
\end{aligned}
$$

We would like to point out the role of $C_{i j 33}^{(3)}$ and $C_{i j 33}^{(1)}$, the Wilson coefficients of the operators $Q_{\text {lequ }}^{(3)}$ and $Q_{\text {lequ }}^{(1)}$ respectively. These operators enter in the running of $C_{e \gamma}^{i j}$ with a coefficient proportional to the Yukawa coupling. Hence we restrict ourselves to the contribution of the top quarks in Eq. (11). Note that $Q_{\text {lequ }}^{(3)}$ mixes directly into $Q_{e \gamma}$, resulting in a rather stringent limit on $C_{i j 33}^{(3)}(\Lambda)$ from $\mathrm{BR}\left(l_{i} \rightarrow l_{j} \gamma\right)$. The constraint on $C_{i j 33}^{(1)}(\Lambda)$ is weaker, since $Q_{\text {lequ }}^{(1)}$ enters only indirectly, i.e. through $Q_{\text {lequ }}^{(3)}$.

In Tables 5 and 6 we list the limits on the various coefficients of Eq. (11) obtained from $\mu \rightarrow e \gamma$ and $\tau \rightarrow \mu \gamma$, respectively. Obviously, very similar limits can be derived for $\tau \rightarrow e \gamma$. The limits are given for several choices of the large scale $\Lambda$. As can be seen from the entries of Tables 5 and 6 , the effect of the RG evolution is very modest for $C_{e \gamma}^{i j}$ and more pronounced for $C_{e Z}^{i j}$. We also point out that the limits obtained for $C_{e Z}^{i j}$ through the effect of the $\mathrm{RG}$ evolution is considerably more stringent than the corresponding limit from a standard NLO calculation, as can be seen by comparing the entries in Tables 3 and 4 with those in Tables 5 and 6, respectively. More important, however, is the fact that without taking the RG into ac- 


\begin{tabular}{||c||c|c|c||}
\hline \hline \multicolumn{4}{|c||}{$\boldsymbol{\mu} \rightarrow \boldsymbol{e} \gamma$} \\
\hline Coeff. & $\Lambda=10^{3} \mathrm{GeV}$ & $\Lambda=10^{5} \mathrm{GeV}$ & $\Lambda=10^{7} \mathrm{GeV}$ \\
\hline$C_{e \gamma}^{21}$ & $2.7 \cdot 10^{-10}$ & $2.9 \cdot 10^{-6}$ & $3.1 \cdot 10^{-2}$ \\
$C_{e Z}^{21}$ & $2.5 \cdot 10^{-8}$ & $1.0 \cdot 10^{-4}$ & $7.1 \cdot 10^{-1}$ \\
$C_{\text {lequ(3) }}^{2133}$ & $3.6 \cdot 10^{-9}$ & $1.4 \cdot 10^{-5}$ & $9.8 \cdot 10^{-2}$ \\
$C_{\text {lequ(1) }}^{2133}$ & $1.9 \cdot 10^{-6}$ & $2.5 \cdot 10^{-3}$ & $\mathrm{n} / \mathrm{a}$ \\
$C_{\text {lequ(3) }}^{2122}$ & $4.8 \cdot 10^{-7}$ & $1.9 \cdot 10^{-3}$ & $\mathrm{n} / \mathrm{a}$ \\
$C_{\text {lequ(1) }}^{2122}$ & $2.6 \cdot 10^{-4}$ & $3.3 \cdot 10^{-1}$ & $\mathrm{n} / \mathrm{a}$ \\
\hline \hline
\end{tabular}

Table 5. Limits on the Wilson coefficients defined at the scale $\lambda=\Lambda$ for three choices of $\Lambda=10^{3}, 10^{5}, 10^{7} \mathrm{GeV}$.

count, it would be impossible to get meaningful limits on the coefficients of $C_{i j 33}^{(3)}(\Lambda)$ and $C_{i j 33}^{(1)}(\Lambda)$. For illustration, we have also included in the Tables limits on these coefficients in the charm sector, $C_{i j 22}^{(3)}(\Lambda)$ and $C_{i j 22}^{(1)}(\Lambda)$. Since the corresponding Yukawa coupling is smaller, the limits are less constraining.

\begin{tabular}{||c||c|c|c||}
\hline \hline \multicolumn{4}{|c||}{$\tau \rightarrow \mu \gamma$} \\
\hline Coeff. & $\Lambda=10^{3} \mathrm{GeV}$ & $\Lambda=10^{4} \mathrm{GeV}$ & $\Lambda=10^{5} \mathrm{GeV}$ \\
\hline$C_{e \gamma}^{32}$ & $3.0 \cdot 10^{-6}$ & $3.1 \cdot 10^{-4}$ & $3.2 \cdot 10^{-2}$ \\
$C_{e Z}^{32}$ & $2.8 \cdot 10^{-4}$ & $1.5 \cdot 10^{-2}$ & $\sim 1.1$ \\
$C_{\text {lequ(3) }}^{3233}$ & $4.0 \cdot 10^{-5}$ & $2.2 \cdot 10^{-3}$ & $1.6 \cdot 10^{-1}$ \\
$C_{\text {lequ(1) }}^{3233}$ & $2.1 \cdot 10^{-2}$ & $5.9 \cdot 10^{-1}$ & $\mathrm{n} / \mathrm{a}$ \\
$C_{\text {lequ(3) }}^{322}$ & $5.4 \cdot 10^{-3}$ & $3.0 \cdot 10^{-1}$ & $\mathrm{n} / \mathrm{a}$ \\
$C_{\text {lequ(1) }}^{3222}$ & $\sim 2.8$ & $\mathrm{n} / \mathrm{a}$ & $\mathrm{n} / \mathrm{a}$ \\
\hline \hline
\end{tabular}

Table 6. Limits on the Wilson coefficients defined at the scale $\lambda=\Lambda$ for three choices of $\Lambda=10^{3}, 10^{4}, 10^{5} \mathrm{GeV}$.

\section{Conclusion}

In these proceedings we have described the first steps towards taking the Standard Model effective field theory, that is the Lagrangian given in Eq. (1), seriously as a quantum field theory. This allows us to perform well defined calculations beyond leading order in the Standard Model couplings and apply renormalisation group techniques. At the current stage, the motivation to go beyond leading order is not to obtain limits on the coefficients that are more precise by $\sim 10 \%$. Rather, performing NLO calculations and taking RG evolution effects into account, we obtain qualitatively new results. This is the case in particular when considering the very stringent limits of low-energy LFV experiments.

We take the attitude that $\Lambda$, the scale of new physics responsible for LFV effects is much larger than the electroweak scale. Hence we restrict ourselves to terms suppressed by at most two powers of $\Lambda$, i.e. to single insertions of Dim-6 operators. Ideally, the coefficients of these operators could all be measured experimentally. This would provide a huge amount of information about the nature of the UV complete theory valid beyond the scale $\Lambda$. In practise, a relatively small number of experiments related to LFV in the charged sector will be carried out at energy scales well below $\Lambda$ and typically result in exclusion limits. In order to extract the maximal possible amount of information from these experiments it is essential to perform computations beyond tree level and take RG evolution into account.

A complete experimental determination of the various coefficients is certainly not a realistic prospect, but even partial information on the coefficients will provide extremely useful guidance for the search of the UV complete theory. However, for this it is essential to know $C_{k}^{(6)}(\Lambda)$, the coefficients at the high scale $\Lambda$. It is at this scale where there is the most direct link between the effective theory and the underlying theory. Even if the underlying theory does not produce an operator $Q_{e \gamma}$ when integrating out the heavy modes it can still result in the LFV decay $\mu \rightarrow e \gamma$. To describe this effect, the inclusion of RG evolution is at least as important as the inclusion of one-loop corrections. In particular, if $\Lambda$ is considered to be far above the electroweak scale, $R G$ evolution leads to qualitatively new effects and can lead to substantial improvements in the limits that are extracted from low-energy measurements.

In the results presented here we have considered the decay $l_{i} \rightarrow l_{j} \gamma$ at NLO and have taken RG effects into account. However, other processes such as muon conversion have been completely ignored and for others, e.g. the decay $l_{i} \rightarrow 3 l_{j}$ only a tree-level analysis has been made. Furthermore, the rather unrealistic assumption has been made that only a single coefficient at a time is non-vanishing. Clearly, there is considerable room for improvement. We are convinced that with a more complete analysis, LFV processes can play an even more important role in the search for physics beyond the Standard Model.

\section{Acknowledgements}

The work of GMP is supported by the Swiss National Science Foundation (SNF) under contract 200021_160156.

\section{References}

[1] Y. Fukuda et al. (Super-Kamiokande), Phys. Rev. Lett. 81, 1562 (1998), hep-ex/9807003

[2] Q.R. Ahmad et al. (SNO), Phys. Rev. Lett. 87, 071301 (2001), nucl-ex/0106015

[3] Q.R. Ahmad et al. (SNO), Phys. Rev. Lett. 89, 011301 (2002), nucl-ex/0204008

[4] A. Crivellin, A. Kokulu, C. Greub, Phys. Rev. D87, 094031 (2013), 1303. 5877

[5] A. Abada, M.E. Krauss, W. Porod, F. Staub, A. Vicente, C. Weiland, JHEP 11, 048 (2014), 1408. 0138

[6] L. Calibbi, P. Paradisi, R. Ziegler, Eur. Phys. J. C74, 3211 (2014), 1408.0754

[7] B. Gripaios, M. Nardecchia, S.A. Renner, JHEP 05, 006 (2015), 1412.1791 
[8] J. Kersten, J.h. Park, D. Stöckinger, L. VelascoSevilla, JHEP 08, 118 (2014), 1405. 2972

[9] M. Beneke, P. Moch, J. Rohrwild (2015), 1508.01705

[10] F. Feruglio, P. Paradisi, A. Pattori (2015), 1509.03241

[11] W. Buchmuller, D. Wyler, Nucl. Phys. B268, 621 (1986)

[12] B. Grzadkowski, M. Iskrzynski, M. Misiak, J. Rosiek, JHEP 10, 085 (2010), 1008 . 4884

[13] L. Lehman, Phys. Rev. D90, 125023 (2014), 1410. 4193

[14] S.T. Petcov, Sov. J. Nucl. Phys. 25, 340 (1977), [Erratum: Yad. Fiz.25,1336(1977)]

[15] P. Minkowski, Phys. Lett. B67, 421 (1977)

[16] S. Weinberg, Phys. Rev. Lett. 43, 1566 (1979)

[17] J. Adam et al. (MEG), Phys. Rev. Lett. 110, 201801 (2013), 1303.0754

[18] U. Bellgardt et al. (SINDRUM), Nucl. Phys. B299, 1 (1988)

[19] A.M. Baldini et al. (2013), 1301.7225

[20] N. Berger (Mu3e), Nucl. Phys. Proc. Suppl. 248-250, 35 (2014)

[21] B. Aubert et al. (BaBar), Phys. Rev. Lett. 104, 021802 (2010), 0908.2381

[22] K. Hayasaka et al., Phys. Lett. B687, 139 (2010), 1001.3221

[23] D. Decamp et al. (ALEPH), Phys. Rept. 216, 253 (1992)

[24] O. Adriani et al. (L3), Phys. Lett. B316, 427 (1993)

[25] R. Akers et al. (OPAL), Z. Phys. C67, 555 (1995)

[26] P. Abreu et al. (DELPHI), Z. Phys. C73, 243 (1997)

[27] G. Aad et al. (ATLAS), Phys. Rev. D90, 072010 (2014), 1408.5774

[28] Tech. Rep. CMS-PAS-EXO-13-005, CERN, Geneva (2015), https://cds. cern. ch/record/2019863

[29] G. Aad et al. (ATLAS), Phys. Lett. B716, 1 (2012), 1207.7214

[30] S. Chatrchyan et al. (CMS), Phys. Lett. B716, 30 (2012), 1207.7235

[31] G. Aad et al. (ATLAS) (2015), 1508.03372

[32] V. Khachatryan et al. (CMS), Phys. Lett. B749, 337 (2015), 1502.07400

[33] R.K. Kutschke (2011), 1112.0242

[34] Y. Kuno (COMET), PTEP 2013, 022 C01 (2013)

[35] W.H. Bertl et al. (SINDRUM II), Eur. Phys. J. C47, 337 (2006)

[36] G.M. Pruna, A. Signer, JHEP 10, 14 (2014), 1408.3565
[37] E.E. Jenkins, A.V. Manohar, M. Trott, JHEP 10, 087 (2013), 1308.2627

[38] E.E. Jenkins, A.V. Manohar, M. Trott, JHEP 01, 035 (2014), 1310.4838

[39] R. Alonso, E.E. Jenkins, A.V. Manohar, M. Trott, JHEP 04, 159 (2014), 1312 . 2014

[40] A. Crivellin, S. Najjari, J. Rosiek, JHEP 04, 167 (2014), 1312.0634

[41] Y. Kuno, Y. Okada, Rev. Mod. Phys. 73, 151 (2001), hep-ph/9909265

[42] K.A. Olive et al. (Particle Data Group), Chin. Phys. C38, 090001 (2014)

[43] M. Bicer et al. (TLEP Design Study Working Group), JHEP 01, 164 (2014), 1308. 6176

[44] A. Abada, V. De Romeri, S. Monteil, J. Orloff, A.M. Teixeira, JHEP 04, 051 (2015), 1412.6322

[45] A.A. Petrov, D.V. Zhuridov, Phys. Rev. D89, 033005 (2014), 1308.6561

[46] A. Crivellin, M. Hoferichter, M. Procura, Phys. Rev. D89, 093024 (2014), 1404 . 7134

[47] S.M. Barr, A. Zee, Phys. Rev. Lett. 65, 21 (1990), [Erratum: Phys. Rev. Lett.65,2920(1990)]

[48] D. Chang, W.S. Hou, W.Y. Keung, Phys. Rev. D48, 217 (1993), hep-ph/9302267

[49] F. Feruglio (2015), 1509.08428

[50] B.M. Dassinger, T. Feldmann, T. Mannel, S. Turczyk, JHEP 10, 039 (2007), 0707. 0988

[51] A. Celis, V. Cirigliano, E. Passemar (2014), 1409.4439

[52] A. Celis, V. Cirigliano, E. Passemar, Phys. Rev. D89, 095014 (2014), 1403. 5781

[53] G. Blankenburg, J. Ellis, G. Isidori, Phys. Lett. B712, 386 (2012), 1202.5704

[54] R. Harnik, J. Kopp, J. Zupan, JHEP 03, 026 (2013), 1209.1397

[55] J. Kopp, M. Nardecchia, JHEP 10, 156 (2014), 1406.5303

[56] D. Aristizabal Sierra, A. Vicente, Phys. Rev. D90, 115004 (2014), 1409.7690

[57] L. de Lima, C.S. Machado, R.D. Matheus, L.A.F. do Prado (2015), 1501.06923

[58] I. Doršner, S. Fajfer, A. Greljo, J.F. Kamenik, N. Košnik, I. Nišandžic, JHEP 06, 108 (2015), 1502.07784

[59] Y. Omura, E. Senaha, K. Tobe, JHEP 05, 028 (2015), 1502.07824

[60] W. Altmannshofer, S. Gori, A.L. Kagan, L. Silvestrini, J. Zupan (2015), 1507.07927

[61] A. Crivellin, G. D’Ambrosio, J. Heeck, Phys. Rev. D91, 075006 (2015), 1503.03477 